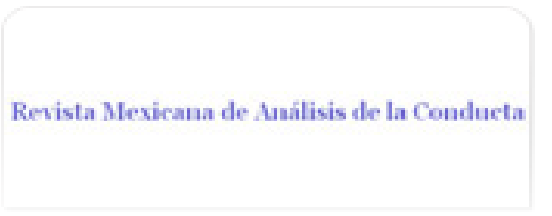

Revista Mexicana de Análisis de la Conducta ISSN: 0185-4534

editora@rmac-mx.org

Sociedad Mexicana de Análisis de la Conducta México

López, Florente; Menez, Marina

Magnitud del reforzador y reforzamiento periódico

Revista Mexicana de Análisis de la Conducta, vol. 34, núm. 1, enero-junio, 2008, pp. 7-23

Sociedad Mexicana de Análisis de la Conducta

Guadalajara, México

Disponible en: http://www.redalyc.org/articulo.oa?id=59311114002

- Cómo citar el artículo

- Número completo

- Más información del artículo

Página de la revista en redalyc.org

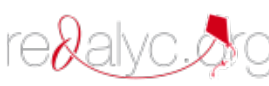

Sistema de Información Científica

Red de Revistas Científicas de América Latina, el Caribe, España y Portugal Proyecto académico sin fines de lucro, desarrollado bajo la iniciativa de acceso abierto 


\title{
MAGNITUD DEL REFORZADOR Y REFORZAMIENTO PERIÓDICO
}

\author{
REINFORCER MAGNITUDE AND PERIODIC REINFORCEMEN) \\ FLORENTE LÓPEZ Y MARINA MENEZ \\ FACULTAD DE PSICOLOGÍA \\ UNIVERSIDAD NACIONAL AUTÓNOMA DE MÉXICO
}

\begin{abstract}
RESUMEN
A fin de evaluar los efectos de la magnitud del reforzador sobre el control temporal de la conducta en programas periódicos, dos grupos de ratas fueron sometidos a programas de Intervalo Fijo de 30 y 90 segundos, la mitad de los animales de cada grupo recibió una pella como reforzador y la otra mitad recibió tres pellas. Se observó que la tasa de respuesta de carrera fue mayor en los grupos con la mayor magnitud, mientras que la tasa global permaneció sin cambios. En varias mediciones del tiempo de inicio al responder, se observó que incrementó con la magnitud de reforzamiento, mientras que la distribución de respuestas en el intervalo asumió una forma sigmoidea para todos los casos. Estos hallazgos son discutidos en el contexto de la hipótesis del doble papel del reforzador y del control temporal de la conducta, como una forma de conducta adaptativa resultante de factores temporales y motivacionales.
\end{abstract}

Palabras clave: Magnitud del reforzador, programas de Intervalo Fijo, control temporal, hipótesis dual del reforzador, ratas.

1. La presente investigación se realizó gracias al financiamiento otorgado por la Universidad Nacional Autónoma de México (DGAPA: PAPIIT-IN301505). Favor de enviar correspondencia a cualquiera de los autores a: Facultad de Psicología, Universidad Nacional Autónoma de México, Avenida Universidad 2004. Col. Copilco-Universidad, 04510 Coyoacán, México D. F., MEXICO o escribir al correo electrónico del primer autor florente@servidor.unam.mx. 


\begin{abstract}
To analyze the effects of reinforcer magnitude on the temporal control of behavior in periodic reinforcement, two groups of rats were exposed to 30 and 90 seconds Fixed Interval schedules. Half of the animals in each group received one pellet as a reinforcer while the other half received three pellets. Local response rates were higher with the larger reinforcer magnitude, but no differences were observed on the global rates. Also, the larger reinforcer magnitude generated longer response latencies, but the within-interval response distribution followed a sigmoid function in all cases. These findings are discussed in the context of the dual role of reinforcement hypothesis and temporal control as an adaptive form of behavior resulting from temporal and motivational factors.
\end{abstract}

Key words: Reinforcement magnitude, Fixed Interval schedules, temporal control, dual role hypothesis, rats.

En la investigación de programas periódicos dos variables que han provocado gran interés en los investigadores son la frecuencia y la magnitud del reforzamiento. Se considera que ambas tienen efectos motivacionales, es decir, sobre el nivel de respuesta, pero que solo la primera está involucrada en la regulación temporal de la conducta. En los programas de reforzamiento de Intervalo Fijo (IF), en los que se programa la entrega de reforzador para la primera respuesta después de un tiempo fijo, la frecuencia se relaciona con el intervalo fijo programado: a mayor el intervalo fijo menor la frecuencia de reforzamiento. En estos programas se han investigado los efectos de la frecuencia de reforzamiento sobre la tasa de respuesta obtenida en dos modalidades: la tasa de respuesta global, determinada sobre el tiempo entre reforzadores (TER), y la tasa de carrera, determinada sobre el tiempo de carrera (tiempo de la $1^{\mathrm{a}}$ a la última respuesta en un intervalo). Los efectos sobre estas variables son claros: a mayor la frecuencia de reforzamiento mayor la tasa de respuesta para ambas determinaciones (López y Menez, 2005). Los efectos de la magnitud de reforzamiento no parecen ser tan claros: algunos investigadores encuentran que la tasa de carrera incrementa conforme aumenta la magnitud (Lowe, Davey y Harzem, 1974; Meltzer y Brahleck, 1968) mientras que otros encuentran que disminuye (Staddon, 1970). De igual manera, algunos investigadores proporcionan evidencia de que la tasa global de respuesta incrementa con incrementos en la magnitud de reforzamiento (Jensen y Fallon, 1973; Stebbings, Mead y Martin, 1959) mientras que otros observan el efecto opuesto (Staddon, 1970), incrementos transitorios (Keesey y Kling, 1961) o ausencia de efectos (Lowe, Davey y Harzem, 1974). En resumen, a diferencia de los efectos de la frecuencia de reforzamiento sobre 
la tasa de respuesta en programas IF, los efectos de la magnitud del reforzador no han sido consistentes de una investigación a otra.

Otros hallazgos de los efectos de la magnitud del reforzador en programas periódicos, importantes desde el punto de vista de la moderna investigación sobre control temporal, son los observados sobre el patrón de respuesta. En este caso se suelen tomar dos indicadores del control: uno relacionado con el tiempo de inicio del responder después del reforzador y otro con la distribución de respuestas entre reforzadores. Respecto al primero, dos estudios encuentran que el tiempo a la primera respuesta en un intervalo, o pausa pos-reforzamiento (PPR), aumenta con la magnitud del reforzador (Lowe, Davey y Harzem, 1974; Staddon, 1970); otro estudio no encuentra efectos (Harzem, Lowe, y Davey, 1975) y uno más encuentra una relación negativa entre magnitud y tamaño de la PPR (Ludvig, Conover y Shizgal, 2007). En otros estudios el tiempo de inicio del responder se determina obteniendo el tiempo desde el reforzador hasta el momento en que la conducta cambia de una tasa baja a un patrón de respuesta alto y relativamente constante. Dos estudios que emplearon este sistema arrojaron resultados completamente contradictorios y, por su interés para los modelos actuales de control y estimación temporal, los describiremos con mayor amplitud.

En una investigación con un programa mixto Intervalo-Fijo 30-s IntervaloFijo 150-s (IF30 IF150) con diferentes respuestas asociadas a cada componente, Blomeley, Lowe y Wearden (2004) manipularon la probabilidad de que estuviera vigente uno u otro valor de IF. Analizaron los datos de la respuesta asociada a IF30 cuando el IF150 estaba vigente, por lo que pudieron obtener las respuestas a la palanca por un periodo mayor a los 30 segundos sin reforzamiento (hasta que el animal se movía hacia la otra palanca al discriminar que la primera ya no sería reforzada). Se acondicionó un rotor para presentar diferentes concentraciones de leche condensada (30\%-10\%-30\%50\%-30\%-70\%, en ese orden). Los tiempos de inicio fueron más breves en las magnitudes grandes que en las bajas, lo que también se manifestó en la PPR promedio: $17.6 \mathrm{~s}$ para la magnitud de $10 \%, 21.8 \mathrm{~s}$ para $30 \%, 28.01 \mathrm{~s}$ para $50 \%$ y 30.9 s para la de $70 \%$. Es decir, la magnitud mayor programada generó un incremento de casi el doble en la PPR que la magnitud menor. Una relación similar, aunque no siempre monótonamente creciente para todas las ratas, se observó entre magnitud del reforzador y tiempo respondiendo en la palanca. En otras palabras, los aumentos en la concentración del reforzador produjeron que las ratas empezaran a responder más tarde en la palanca IF30 y que permanecieran más tiempo en esa palanca. Por otra parte, a fin de describir la distribución de respuestas, obtuvieron las tasas de respuesta promedio en intervalos sucesivos de 3-s a partir del reforzador y ajustaron la distribución normal. Los ajustes fueron satisfactorios y se observó que el pico de la curva se desplazó hacia la derecha conforme aumentó la concentra- 
ción del reforzador, mientras que la altura del pico se mantuvo relativamente constante y el coeficiente de variación disminuyó con los incrementos en la magnitud.

En otro estudio Ludvig, Conover, y Shizgal (2007) evaluaron la relación entre estimación temporal y magnitud del reforzador aplicando un procedimiento de pico. En este procedimiento es posible determinar la distribución de respuestas en el tiempo, el tiempo de inicio o momento de transición de una tasa baja a una alta tasa de respuesta, y el tiempo de terminación o momento de transición de una tasa alta a una tasa baja de respuesta. A fin de manipular la magnitud se utilizaron niveles bajos o altos de estimulación cerebral aplicada a ratas. Las ratas hicieron pausas más largas y mayores tiempos de inicio con el reforzador de baja que con el de alta magnitud. En el ajuste de una función Gaussiana, observaron que los picos de la función ocurrieron antes en la magnitud menor que en la mayor. En las mismas funciones, así como en el análisis de intervalos individuales, encontraron un gran efecto de la estimulación cerebral en los tiempos de inicio y ausencia de efecto en los tiempos de terminación. Los hallazgos contradicen varias teorías de estimación temporal, que no predicen efectos de la manipulación crónica de la magnitud de reforzamiento.

Los autores del estudio anterior sugirieron una posible explicación de los contradictorios efectos encontrados en los diversos experimentos. Consideran que la magnitud del reforzador puede influir la estimación temporal de dos maneras: Primero, por medio de un pos-efecto inmediato del reforzador que demora el responder de tal forma que a mayor la magnitud mayor será la demora. Segundo, por medio de un efecto anticipatorio del reforzador de tal forma que a mayor la magnitud, mayor anticipación se producirá y más pronto responderá el animal. Estos efectos tenderán a predominar según la forma de programación del programa de intervalo. En el caso de las dos investigaciones arriba revisadas, se utilizaron diferentes procedimientos temporales de entrega del reforzador. Blomeley y cols. (2004) iniciaban el intervalo con la entrega del reforzador, mientras que Ludvig y cols. (2007) emplearon un estímulo visual como marcador temporal. De esta manera los efectos postreforzador estuvieron presentes en el primer pero no en el segundo estudio en el que, debido a que el marcador temporal se asociaba con magnitudes distintas, predominó el efecto anticipatorio.

Desde luego existen otros factores que pueden dar una idea más completa de las diferencias en resultados entre estudios. Por ejemplo, el tamaño del intervalo empleado, la operación para manipular magnitud, la naturaleza del diseño de comparación de magnitudes (intra sesión o entre condiciones) y el número de sesiones en que se obtuvieron las comparaciones. La combinación de estos posibles factores en un solo estudio no parece una solución práctica para resolver la naturaleza de las diferencias. En el presente estu- 
dio seguimos la estrategia de evaluar de manera sistemática los efectos de magnitud en condiciones que permitan verificar la importancia relativa del tamaño del intervalo y el momento de la evaluación (número de sesión), en condiciones en que el intervalo es señalado por el reforzador. Se aplicaron dos valores de intervalo, uno corto de 30 segundos y otro largo de 90 segundos. Para determinar si algunos efectos encontrados en otros estudios solo son de transición, los programas de IF se mantuvieron por un número relativamente prolongado de sesiones y se tomaron mediciones sesión a sesión. Finalmente, cada valor de IF se programó en grupos con diferentes magnitudes, intentando que estas tuvieran un contraste razonable, a fin de determinar tanto los efectos crónicos del programa temporal como los cambios ocurridos a lo largo del experimento.

\section{MÉTODO}

\section{Sujetos}

Doce ratas Wistar macho, criadas en el bioterio de la facultad, de aproximadamente 3 meses de edad al inicio del experimento y mantenidas al $80 \%$ de su peso ad libitum. Excepto durante las sesiones experimentales, las ratas estuvieron alojadas en jaulas individuales en el bioterio, con agua continuamente disponible, en un ciclo de luz-oscuridad de 12 horas.

\section{Aparatos}

Siete cajas de condicionamiento operante, de $21.0 \mathrm{~cm}$ de altura $\times 30.5 \mathrm{~cm}$ de largo $\times 24.1 \mathrm{~cm}$ de ancho, marca MED-Associates. La pared frontal de cada caja estaba provista de una palanca, una luz indicadora y un orificio de acceso a las pellas entregadas por un dispensador rotatorio, colocado en la parte central de la pared y situado a $5 \mathrm{~cm}$ de la rejilla sobre la que se posaba el animal. Además de la luz ubicada sobre la palanca, se colocó otra en el orificio del dispensador de pellas. Otra luz colocada en la pared superior trasera de la caja, proporcionó la iluminación general. Se utilizaron pellas marca Noyes de $45 \mathrm{mg}$ como reforzador. Se adaptó una fuente de ruido blanco para enmascarar ruidos externos, además de que las cajas de prueba se mantuvieron aisladas de luz y ruido externos al estar colocadas dentro de una caja sono-amortiguada. Una computadora personal provista de una interfase MED, controló los eventos y registró los tiempos de ocurrencia de las respuestas y lo reforzadores con una aproximación a la centésima de segundo. 


\section{PROCEDIMIENTO}

\section{Preentrenamiento}

Todas las ratas fueron moldeadas manualmente a presionar la palanca. Al inicio de la sesión, se encendieron la luz general y la luz colocada arriba de la palanca. Cuando la rata husmeaba, tocaba o presionaba la palanca, estas luces se apagaban y se activaba el dispensador de pellas, al mismo tiempo que se encendía la luz del orificio del comedero. Después de tres segundos, el ciclo se reiniciaba. Las ratas tardaron entre dos y tres sesiones en adquirir la respuesta de presionar la palanca. Las sesiones de moldeamiento y las experimentales concluyeron a la entrega del reforzador número 36 . Después de adquirir la respuesta de apretar la palanca, las ratas fueron mantenidas en un programa de Razón Fija 1 por tres sesiones.

\section{Fase experimental}

Al concluir el preentrenamiento, las ratas fueron asignadas aleatoriamente a uno de cuatro grupos de 3 ratas cada uno. Los grupos resultaron de un diseño factorial con Intervalo y Magnitud como factores. El factor intervalo se integró por dos condiciones: Intervalo Fijo 30-s (IF30) e intervalo Fijo 90-s (IF90). EI factor Magnitud se integró por dos condiciones: 1 pella (M1) y 3 pellas. (M3). De esta forma, los grupos del experimento fueron IF30M1, IF30M3, IF90M1 e IF90M3. En estos programas la primera respuesta después del tiempo especificado recibió 1 o 3 reforzadores según el grupo correspondiente. El tiempo se contó a partir de la terminación de los estímulos asociados con el reforzador, excepto para el primer intervalo que empezó con el inicio de la sesión. La entrega del o los reforzadores fue acompañada por un periodo de $3 \mathrm{~s}$ con la luz de la cámara e indicadora apagadas y la luz del comedero encendida. Al concluir este periodo se re-establecieron las condiciones del ensayo con las luces de la cámara y la indicadora encendidas y la del comedero apagada. Las condiciones de cada grupo se mantuvieron vigentes por 60 sesiones.

\section{RESULTADOS}

Las comparaciones se efectuaron tanto en variables relacionadas con fortaleza de la respuesta, normalmente vinculadas con efectos motivacionales, como en la relacionadas con patrón de respuesta, normalmente asociadas con control o estimación temporal.

\section{Tasa de Respuesta}

Se obtuvieron tres indicadores de la fortaleza de la respuesta: (a) La tasa global, calculada como el total de respuestas en una sesión dividido por la 
duración de la sesión menos el tiempo acumulado por la duración de los estímulos asociados a la entrega del reforzador. (b) La tasa terminal calculada como el total de respuestas en una sesión dividido por el tiempo de trabajo o tiempo de la primera respuesta a la entrega del reforzador y (c) El número de respuestas por reforzador.

En la Figura 1 se presentan diagramas de caja obtenidos con las tasas de respuesta globales de todos los sujetos de cada grupo. Los diagramas se construyeron con los datos de cinco sesiones iniciales (1-5), cinco intermedias (26-30) y cinco finales (56-60) de cada sujeto en cada condición. Los diagramas representan los cuartiles 25 y 75 (extremos inferior y superior de la caja, respectivamente), la mediana (símbolo en el interior de la caja) y valores extremos. Puede observarse que al introducir el programa IF (primeras cinco sesiones) existe una tendencia del IF 30-s a generar tasas más elevadas que las de IF 90-s y que, en el primero, la menor magnitud tendió a producir tasas mas altas que la mayor magnitud, mientras que en el segundo no se observaron estas diferencias. Aunque el efecto del valor del IF persistió hasta las sesiones intermedias, esto no ocurrió con las diferencias atribuibles a magnitud en las primeras sesiones. En las últimas cinco sesiones, el patrón de resultados anterior se mantuvo, pero las diferencias atribuibles al valor del intervalo fueron aún menos claras debido a que incrementó la variabilidad de los datos y se generó mayor traslapamiento entre las cajas.

La Figura 2 está construida de manera similar a la anterior, pero en este caso se utilizaron los datos de tasa de carrera de cada sesión de cada sujeto. En general se observa que al introducir los programas IF (primeras cinco sesiones) prácticamente no hubieron diferencias entre los cuatro grupos. Sin embargo, para las sesiones intermedias se observaron mayores tasas en el IF30 que en el IF90 y en la mayor que en la menor magnitud, aunque esto fue menos contundente en la comparación de magnitudes de los grupos sometidos a IF30 (IF30-M1 vs. IF30-M3). Aunque el volumen general de las tasas tendió a ser más elevado, el patrón de resultados anterior persistió en las sesiones finales: una clara tendencia de los programas de IF30 a generar tasas de carrera más elevadas que los de IF90 y una tasa de respuesta mayor en la magnitud alta que en la baja, diferencia que fue más pronunciada en IF90.

En la Figura 3 se presenta la media de respuestas por intervalo, por sesión, para cada grupo. La gráfica sugiere una tendencia del IF90 a generar un mayor número de respuestas que el IF30, aunque la gran variabilidad que se presentó en el grupo IF90M3 hace menos nítida esta diferencia. El número de respuestas por intervalo en cada sesión no sugiere predominio de alguna de las magnitudes en cada valor de IF. Por último, las respuestas por intervalo en cada grupo mostraron muy poca tendencia a cambiar a lo largo de las sesiones, aunque si se observó una moderada variabilidad, mayor para los grupos sometidos a IF90. 
Patrón de respuesta

Para describir el patrón de respuesta se seleccionaron tres mediciones. La pausa pos-reforzamiento o tiempo desde la terminación de los estímulos asociados al reforzador hasta la ocurrencia de la primera respuesta en el intervalo. La vida cuartilar o tiempo empleado para completar un cuarto del total de respuestas en el intervalo. La tasa de respuesta en función del tiempo desde el reforzador.

En la Figura 4 se presenta la pausa pos reforzamiento promediada por sesión, según las descripciones de diagramas de caja antes empleados, y en las sesiones iniciales, intermedias y finales antes descritas. Durante las primeras sesiones se observa que las pausas tienden a ser mayores en IF90 que en IF30 y que, en ambos casos, la magnitud de reforzamiento mayor generó distribuciones con una mayor tendencia hacia valores más altos. Ambas tendencias se acentuaron en las sesiones intermedias y se mantuvieron de esa forma en las sesiones finales.

En la Figura 4 se presenta la vida cuartilar obtenida por cada sujeto de cada grupo en cada una de las sesiones del experimento. La línea continua representa la línea de mejor ajuste de la función Weibull de tres parámetros. Esta función es una función de crecimiento de uso general y que permite resumir apropiadamente tres aspectos importantes de la adquisición, en este caso del control temporal: el tiempo que toma o latencia, qué tan abrupto es el cambio hacia el nivel asintótico y el nivel asintótico (Gallistel, Fairhurst y Balsam, 2004). Los ajustes de esta función a los datos del presente experimento siempre fueron mayores de $85 \%$. En general se observa que todos los grupos presentan un rápido incremento de la vida cuartilar y una tendencia a estabilizarse aproximadamente a las 20 sesiones. Los valores obtenidos en las últimas sesiones se estabilizaron a valores mayores para IF90 que para IF30 y para la magnitud de reforzamiento mayor que para la menor en cada valor de IF.

Las Figuras 5 y 6 muestran los gradientes temporales de cada sujeto. Para obtenerlos se obtuvieron las tasas de respuesta en 15 bins (de tamaño $t / 15$, donde $t$ es el valor temporal del IF) a partir del reforzador hasta el momento de la disponibilidad del mismo y se promediaron sobre las cinco últimas sesiones. Los gradientes se relativizaron respecto a la máxima tasa de respuesta y respecto al valor programado del intervalo, ambos fijados a 1.0. Para una mejor comparación, se ajustó a los datos la siguiente función sigmoidea:

$$
y=\frac{c}{1+e^{-(x-a) / b}}
$$


Donde a estima el centro o tiempo requerido para obtener la mitad del punto máximo de respuestas de la función, $b$ es un estimado de la escala de la función que suele considerarse como un indicador de la precisión de la estimación y $c$ es un estimado del máximo de respuestas de la función.

En la Figura 5 se presentan los datos de las ratas sometidas a IF30 en el grupo que recibió 1 pella (M1, columna izquierda) y el que recibió 3 pellas (M3, columna derecha). La línea continua es la línea de mejor ajuste a la función sigmoidea y en el recuadro se presentan los parámetros de ajuste. Como se puede ver, todos los casos fueron bien representados por la función sigmoidea y puede apreciarse que la mayor magnitud de reforzamiento tendió a producir un desplazamiento del Centro a la derecha de la función y a producir una mayor precisión de la estimación (valores menores en la escala). En la Figura 6 se presentan los datos de las ratas sometidas a IF90 en el grupo que recibió 1 pella (M1, columna izquierda) y el que recibió 3 pellas (M3, columna derecha). Los efectos de magnitud fueron similares a los anteriores: desplazamiento del Centro a la derecha y mayor precisión en la estimación en el grupo que recibió mayor magnitud que el que recibió menor magnitud de reforzamiento, aunque en este caso tales efectos fueron más claros.

En general todos las ratas mostraron un patrón creciente de respuestas, preponderando el patrón negativamente acelerado, aunque el inició de respuestas fue generalmente más tardío en los grupos con mayor magnitud de reforzamiento y en éstos la tasa de crecimiento a su valor máximo fue más pronunciada.

\section{CONCLUSIONES}

Nuestro objetivo en la investigación presentada fue evaluar los efectos de la magnitud de reforzamiento sobre la ejecución y la regulación temporal en programas periódicos. En cuanto a ejecución, por lo general se asume que la tasa de respuesta incrementa en función de la magnitud de reforzamiento. Esta relación se ha demostrado con claridad en programas donde el reforzador es programado a intervalos variables, pero en los programas de intervalo fijo existe la complicación adicional de determinar la base temporal apropiada para obtener la tasa de respuesta. El problema radica en que estos programas generan periodos sin respuesta substanciales después del reforzador y se debe decidir si se incluyen o no como parte del tiempo disponible de respuesta. Por consiguiente, los investigadores han optado por presentar mediciones que incluyen y no incluyen dicho periodo sin respuesta, denominados tasa global y tasa de carrera, respectivamente. En cuanto a los efectos de la magnitud de reforzamiento sobre la tasa global de respuestas aquí solo 
encontramos efectos transitorios. A diferencia del presente, estudios anteriores reportan una relación positiva entre la tasa global de respuestas y la magnitud de reforzamiento (Jensen y Fallon (1973); Stebbings, Mead, y Martin (1959). Esta aparente discrepancia probablemente puede entenderse en términos del número de sesiones empleado. Los citados autores emplearon un número relativamente pequeño de sesiones para evaluar los efectos de magnitud (la mayoría de las veces 10), y nuestros datos muestran que para la sesión 25 prácticamente ya no hubo diferencias, como las que podrían sugerir los datos de las primeras 5 sesiones, particularmente en el IF90 (ver Figura 1).

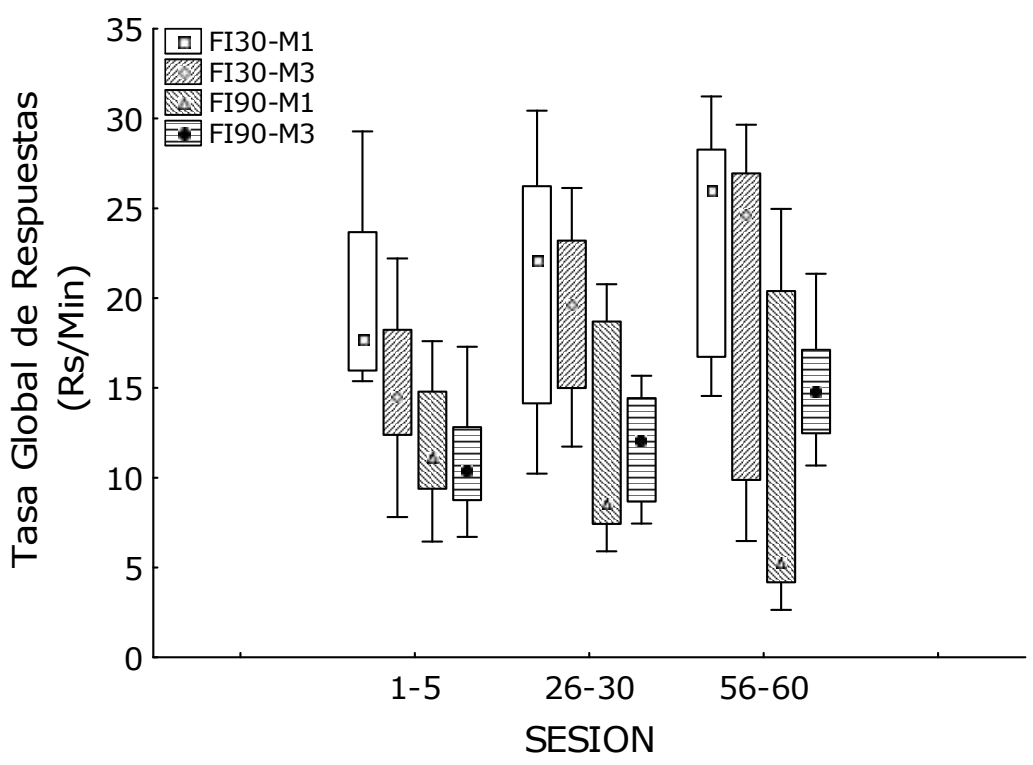

Figura 1.

Por otro lado, los datos del presente experimento sugieren una relación positiva entre la magnitud del reforzamiento y la tasa terminal de respuestas, que se manifiesta con mayor claridad conforme pasan las sesiones pero que prácticamente no ocurre a la introducción del IF. Esto es consistente con los hallazgos de Meltzer y Brahlek (1968) y de Lowe, Davey, y Harzem (1974), quienes reportan tales efectos en las sesiones estables finales. 


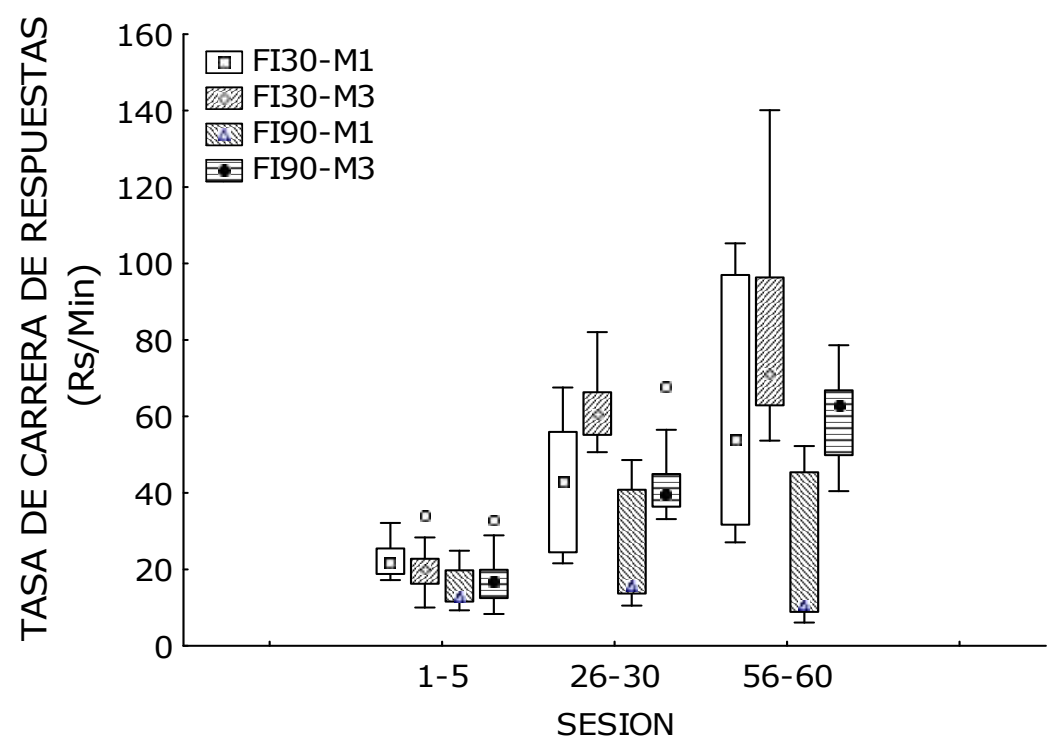

Figura 2.

Los hallazgos anteriores sugieren relaciones un tanto sorprendentes: mientras que los efectos de la magnitud sobre la tasa de respuesta global si acaso se presentan ligeramente en las primeras sesiones y desaparecen conforme transcurren las sesiones, los efectos sobre la tasa de carrera no ocurren en las primeras sesiones sino que se presentan conforme pasan las sesiones. Una forma en que lo anterior podría ocurrir es si (1) el numero de respuestas por intervalo se mantiene relativamente constante a lo largo de las sesiones (2) el número de respuestas es aproximadamente el mismo en cada magnitud de reforzamiento (3) la PPR generada por la magnitud mayor tiende a crecer a valores más altos que para la menor. En estas condiciones, en la magnitud mayor la base temporal será cada vez menor a la de la magnitud menor y, por consiguiente, se obtendrán valores cada vez más altos de la tasa de carrera. Por el contrario, la tasa de respuesta global solo sufrirá incrementos mientras el número de respuestas por intervalo sea mayor para la mayor magnitud, pero si la diferencia en número de respuestas se mantiene constante o solo sufre incrementos en las primeras sesiones, las tasas globales tenderán a ser iguales en las sesiones finales puesto que la base temporal se mantiene fija. Los datos de número de respuestas por intervalo y de PPR del presente experimento se aproximan al patrón descrito (ver Figura 3). 


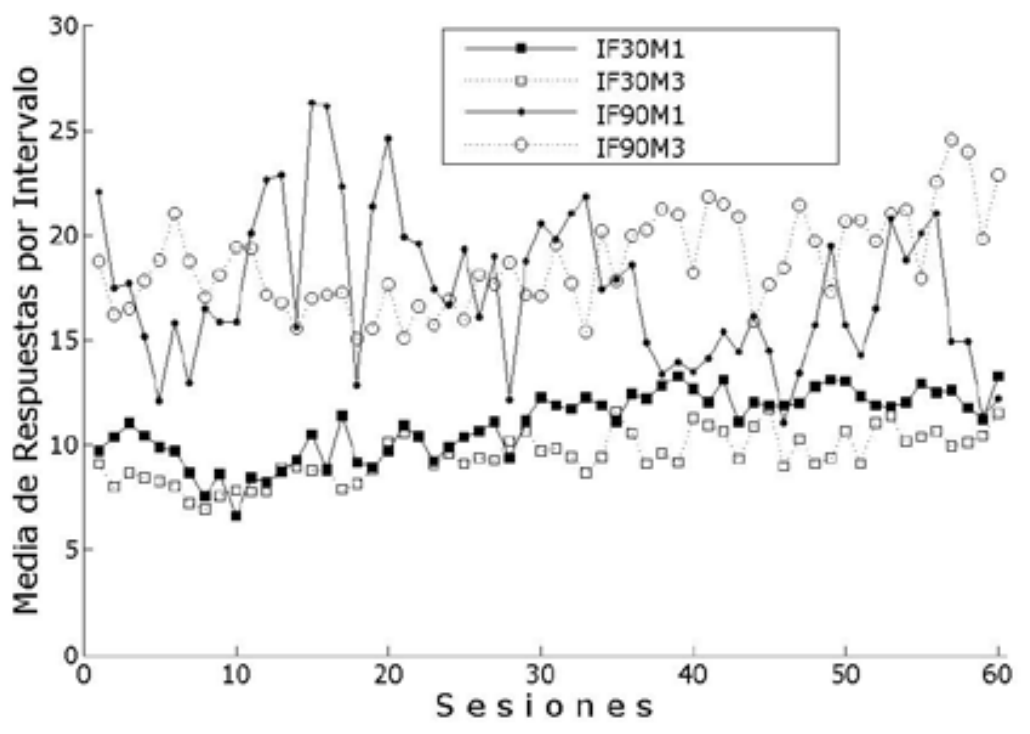

Figura 3.

Las implicaciones más importantes del presente estudio se refieren a los efectos de la magnitud sobre la regulación temporal de la conducta. De particular interés es que la magnitud mostró una relación positiva con el tiempo de inicio de responder dentro del intervalo, lo que pudo comprobarse por medio de tres indicadores: la PPR (Figura 4), la vida cuartilar (Figura 5) y el parámetro a (centro) del ajuste sigmoideo (Figuras 6 y 7 ). Los tres arrojaron valores más elevados en la magnitud mayor que en la menor, por tanto sugiriendo un desplazamiento del inicio del responder hacia valores temporales más elevados con la mayor magnitud. Las mediciones anteriores se relacionan con el momento en que el animal cambia su conducta de un periodo con pocas o ninguna respuesta a un periodo en que se observa una tasa de respuestas relativamente constante. Los efectos aquí observados son congruentes con los obtenidos por Lowe, Davey, y Harzem (1974); Staddon (1970) y Blomeley y cols. (2004). No obstante, es muy importante revisar las condiciones específicas en que se han evaluado los efectos de magnitud. En particular, los efectos aquí reportados se observaron desde aproximadamente la sesión 15 y se mantuvieron hasta la última sesión (ver Figuras 4, 5, 6 y 7). Sin embargo, otros estudios reportan que bajo condiciones de estabilidad con un número de sesiones relativamente grande, no se presentan diferencias en 


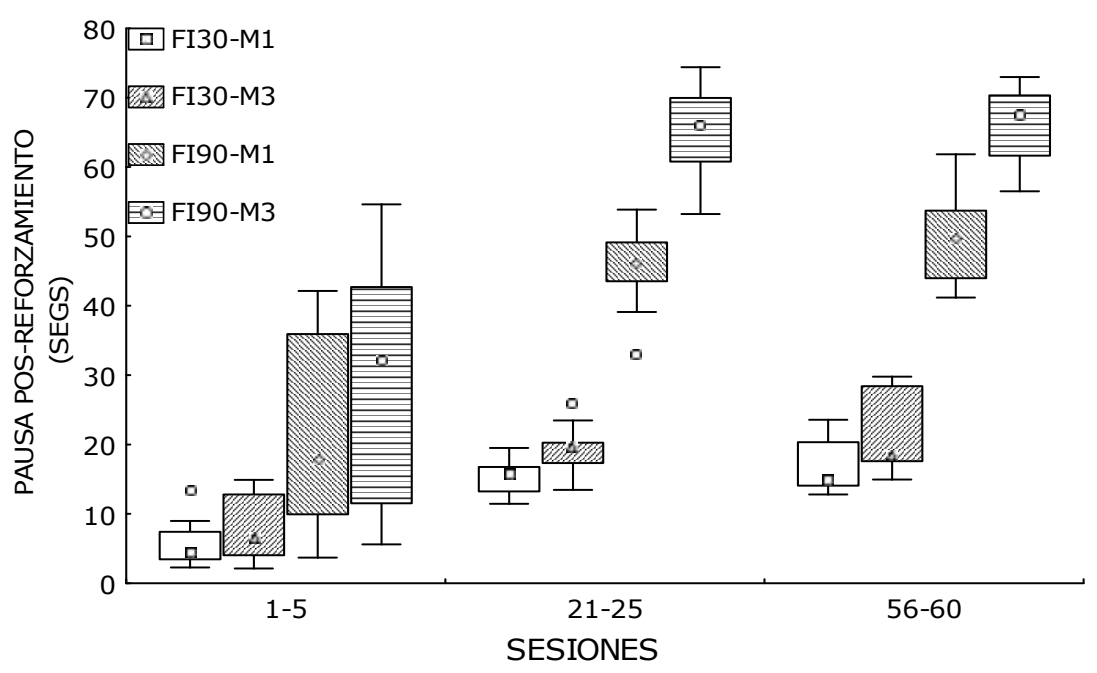

Figura 4.
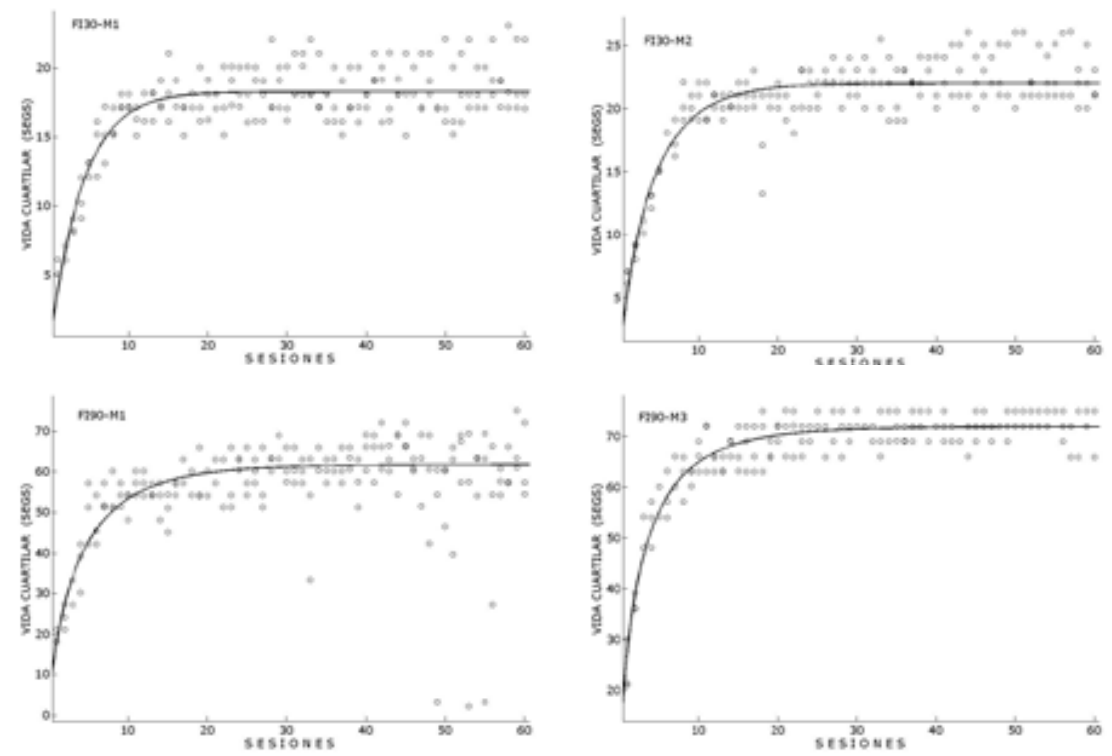

Figura 5. 


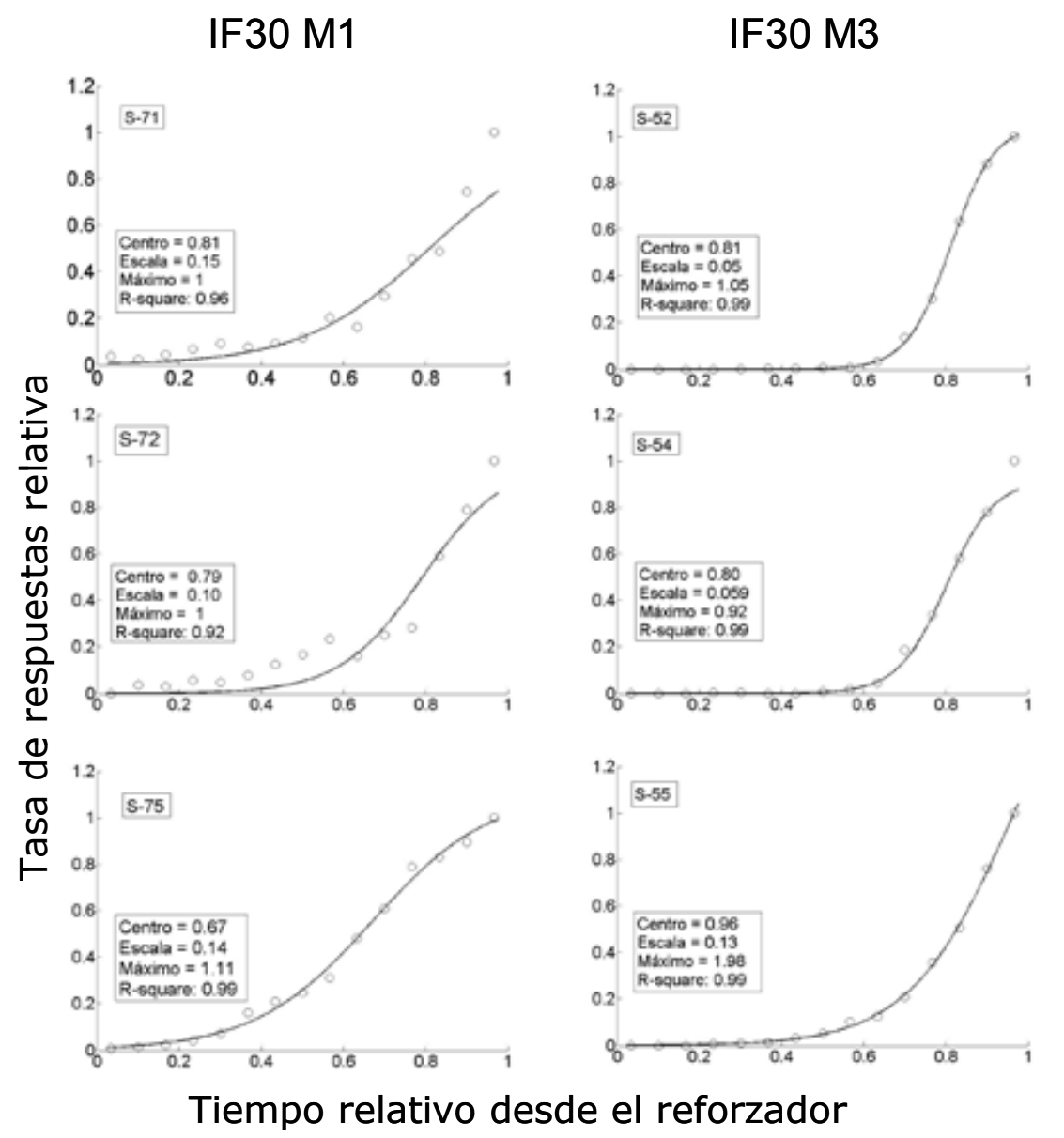

Figura 6. 

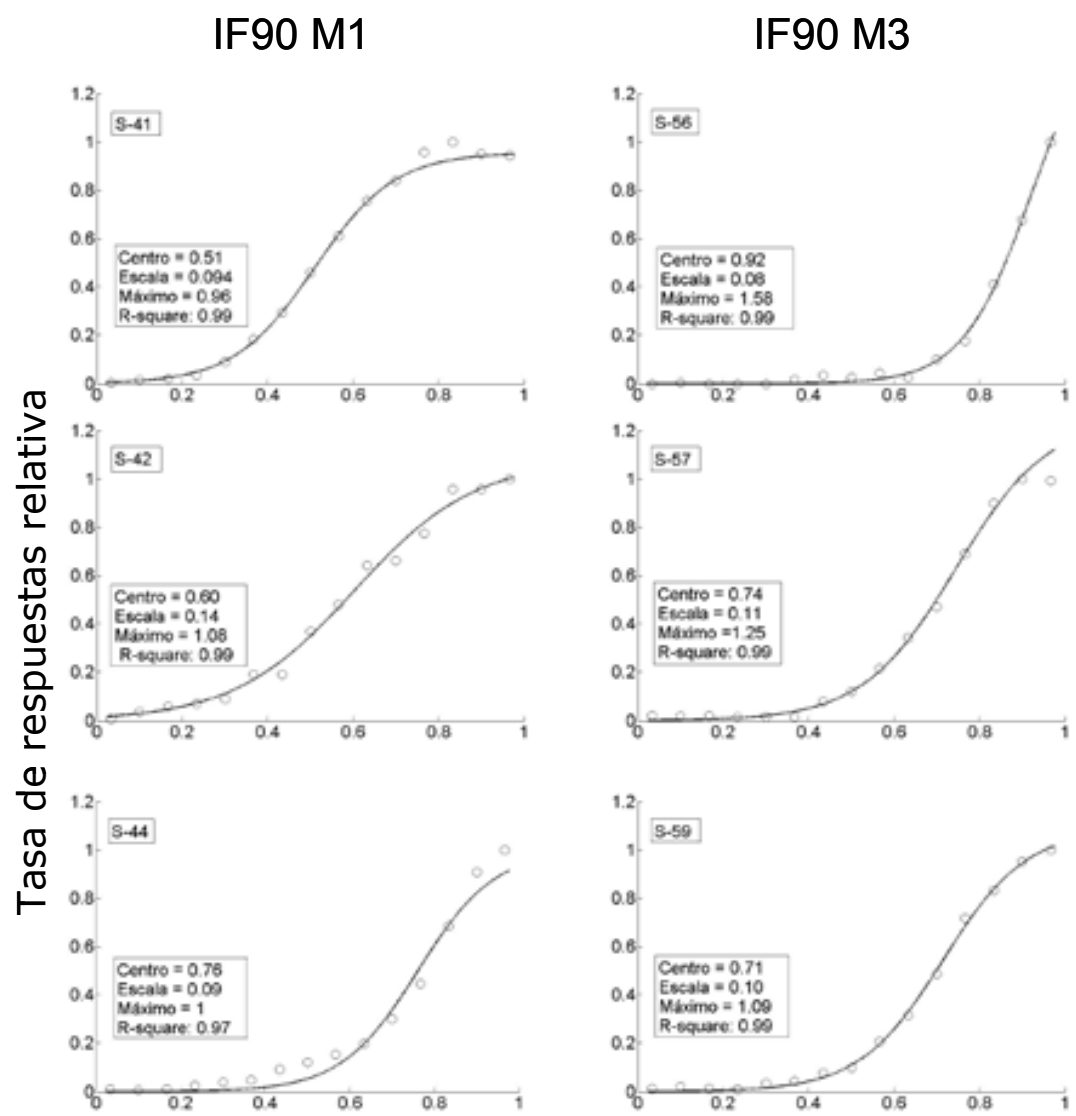

Tiempo relativo desde el reforzador

Figura 7. 
medidas de inicio del responder debidas a magnitud (Jensen y Fallon, 1973; Harzem, Lowe, y Davey, 1975). Aun más, Ludwig y cols. (2007) encontraron que con procedimiento de pico en los ensayos no reforzados, la mayor magnitud generaba inicios del responder más breves que la menor magnitud. A fin de explicar las diferencias entre experimentos dichos autores recurrieron a lo que denominaron la hipótesis del doble papel del reforzador. Según esta hipótesis, se supone que el post-efecto inhibidor del reforzador es mayor mientras mayor es la magnitud $y$, por consiguiente, produce tiempos de inicio mayores. Por otro lado, se considera que mientras mayor es la magnitud, mayor es la anticipación del reforzador generada y, por consiguiente, el tiempo de inicio tenderá a disminuir. En los programas IF estos dos efectos tienden a equilibrarse conforme pasan las sesiones, por lo que no se observarían efectos de la magnitud del reforzador en estado estable. No obstante, en el procedimiento de pico, en que el tiempo del intervalo se inicia por la presentación de un estímulo y no de un reforzador, sólo se presentan los efectos anticipatorios por lo que las magnitudes mayores tienden a producir tiempos de inicio más breves.

La hipótesis del doble papel del reforzador permite explicar los hallazgos de Ludvig y cols (2007), y en cierta forma explican los resultados de Jensen y Fallon (1973) y de Harzem, Lowe, y Davey (1975). Sin embargo, es relativamente contradictoria con los hallazgos del presente estudio y con los de Blomeley y cols. (2004) en los que se observa la aparición de una relación positiva entre magnitud reforzamiento y mediciones del inicio del responder y en ambos casos el inicio del intervalo es indicado por el propio reforzador. En particular, en el presente estudio no se esperaría que en las sesiones finales existieran diferencias debidas a magnitud en las mediciones de inicio de responder, puesto que los efectos discriminativos y anticipatorios del reforzador se habrían equilibrado. Conviene señalar que en este estudio las diferencias en las mediciones de inicio de responder debidas a magnitud, fueron más claras cuando el valor del IF fue de 90 segundos. Cabe la posibilidad entonces de que, conforme aumenta el valor del intervalo, los post-efectos inhibidores del reforzador predominen sobre los anticipatorios, generando así las diferencias observadas. En otras palabras, parece necesario incorporar a la hipótesis del doble papel del reforzador una clara noción sobre el comportamiento de los parámetros inhibitorios y de anticipación en relación a la magnitud. No obstante, para ello se requiere un diseño más poderoso que el presente para evaluar los efectos de interacción entre valor del IF y magnitud.

Como ha ocurrido en otros estudios con programas de intervalo fijo, la curva de respuesta es bien descrita por una función sigmoidea que refleja el control temporal del programa. Las variables motivacionales, como la aquí investigada, no parecen afectar mayormente el control temporal de la conducta sino únicamente sus parámetros indicativos de inicio de respuesta, sensibili- 
dad o tasa máxima de respuesta. El estudio de estos factores permitirá una mejor comprensión del comportamiento ante programas de intervalo como conducta adaptativa en la que se integran el aprendizaje de las propiedades temporales del ambiente con las restricciones motivacionales del mismo.

\section{REFERENCIAS}

Blomeley, F. J., Lowe, C.F., \& Wearden, J.H. (2004). Reinforcer concentration effects on a fixed-interval schedule. Behavioural Processes, 67, 55-66.

Gallistel, C. R., Fairhurst, S., \& Balsam, P. (2004). The learning curve: Implications of a quantitative analysis. Proceedings of the National Academy of Sciences of the United States of America, 101, 13124-13131.

Harzem, P., Lowe, C. F., \& Davey, G. C. L. (1975). After-effects of reinforcement magnitude: Dependence upon context. Quarterly Journal of Experimental Psychology, 27, 579-584.

Jensen, C., \& Fallon, D. (1973). Behavioral after-effects of reinforcement and its omission as a function of reinforcement magnitude. Journal of the Experimental Analysis of Behavior, 19, 459-468.

Keesey, R. E. \& Kling, J. W. (1961). Amount of reinforcement and free-operant responding. Journal of the Experimental Analysis of Behavior, 4, 125-132.

López, F. y Menez, M. (2005) Effects of reinforcement history on response rate and response pattern in periodic reinforcement. Journal of the Experimental Análysis of Behavior, 83, 221-241.

Lowe, C. F., Davey, G. C. L., \& Harzem, P. (1974). Effects of reinforcement magnitude on interval and ratio schedules. Journal of the Experimental Analysis of Behavior, 22, 553-560.

Ludvig E.; Conover; K. and Shizgal, P. (2007). The effects of reinforcer magnitude on timing in rats. Journal of the Experimental Analysis of Behavior, 87, 201-218

Meltzer, D., \& Brahlek, J. A. (1968). Quantity of reinforcement and fixed-interval performance: Within subject effects. Psychonomic Science, 20, 30-31.

Staddon, J. E. R. (1970). Effect of reinforcement duration on fixed-interval responding. Journal of the Experimental Analysis of Behavior, 13, 9-11.

Stebbins, W. C., Mead, P. B., \& Martin, J. M. (1959). The relation of amount of reinforcement to performance under a fixed-interval schedule. Journal of the Experimental Analysis of Behavior, 2, 351-355. 